\title{
Ist eine transforaminale Kortisoninjektion wirklich sinnvoll?
}

Bandscheibenvorfälle mit Wurzelkompression sind eine mögliche Indikation für transforaminale Steroidinjektionen. Sie haben einen kurzfristigen Effekt und führen zu schneller Remobilisierung. Für die Praxis relevant wäre es zu wissen, welche Patienten davon nicht profitieren.

$\mathrm{m}$ Rahmen einer Studie wurden die Daten von 51 Patienten unter anderem mit Radikulopathie, Diskusprolaps, lateralem Rezessus oder auch Sequester im Bereich von L3/4/5 und S1 untersucht. Die Ansprechraten für die Kortikoidinjektionen lagen bei $56 \%$ nach zwei Wochen, $44 \%$ nach drei Monaten und $38 \%$ nach sechs Monaten. Das Outcome nahm ab, wenn mehr als zwei der Risikofaktoren - kombinierter motorischer und sensorischer Defekt, Übergewicht oder degenerative Spinalkanalstenose vorlagen. Die Chance eines Therapieversagens wurde dadurch signifikant erhöht.

Die Ergebnisse der vorgestellten Untersuchung legen nahe, dass Patienten mit einem kombinierten sensorischen und motorischen Defizit frühzeitig an einen Neurochirurgen überwiesen werden sollten, statt sie erst mittels Injektionen zu behandeln.

Würde man die Patientengruppe selektionieren, die am wenigsten von einer Injektion profitiert, würde das die Anzahl unnötiger Prozeduren reduzieren. Gleichzeitig würde man diejenigen finden, die von einer frühen chirurgischen Intervention Vorteile hätten.

Dr. Henrike Ottenjann

Rocher L. Predictive factors of efficacy of transforaminal corticosteroid injections in patients with low back pain and radiculopathy. $9^{\text {th }}$ Interdisciplinary World Congress on Low Back Pain, Singapur, 3. November 2016

\section{Eiskunstläuferin mit Hüftschmerzen}

Erhöhte körperliche Aktivität wie bei Leistungssportlern steigert auf Dauer das Risiko für Schmerzen und Arthrose der Gelenke. Eine individuelle physikalische Therapie kann dabei helfen, nach einem operativen Eingriff den Leistungssport wieder aufzunehmen.

abrumläsionen im Bereich der Hüfte - schränken die Bewegung ein und erhöhen langfristig das Arthroserisiko. Ursachen sind körperliche Aktivitäten, besonders bei Sportarten mit wiederholenden Bewegungen. Sie können aber auch Folge von Verletzungen oder Krankheiten sein, die den Knochen oder Knorpel des Hüftgelenks in Mitleidenschaft ziehen. Die Behandlungsmöglichkeiten sind physikalische Therapie, Injektionen, entzündungshemmende Medikamente oder auch die arthroskopische Naht der Gelenklippe, um eine Hüftarthrose effektiv zu verhindern. Was fehlt, sind Daten zur postoperativen Versorgung. Professor Lynette KhooSummers, Washington University Physicians St. Louis Children's Hospital, USA, stellte in diesem Zusammenhang den besonderen Fall einer Eiskunstläuferin vor, die bei bekannter Hüftdysplasie und Psoriasisarthritis Schmerzen im Bereich beider Hüften hatte.

Die Sportlerin kam wegen eines „femoral anterior glide"-Syndromes und Hüftadduktion mit medialer Rotation zur dritten Hüftoperation in die Klinik. Ziel war, dass die Patientin postoperativ weiter Schlittschuhlaufen kann. Daher folgte der Diagnose angepasst, im Anschluss an die Operation eine individuell physikalische Therapie. Diese beinhaltete Übungen zu korrekter Hüftadduktion, medialer Rotation und Vorwärtsgleiten des Oberschenkels. Gleichzeitig erfolgte eine Stärkung der M. iliopsoas, gluteus maximus, gluteus medius und der tiefen lateralen Hüftrotatoren. Stretching wurde bewusst vermieden. Diese Übungen führten zum Erfolg und könnten ein Anhaltspunkt dafür sein, wie individuelles Training Stärke und Stabilität wieder herstellen kann, um sogar wieder Leistungssport betreiben zu können.

Dr. Henrike Ottenjann

Khoo-Summers L. Examination and treatment of a competitive figure skater with bilateral hip pain and psoriatic arthritis. Washington University School of Medicine St. Louis, Missouri, USA. Physical Therapy and Department of Orthopaedic Surgery. $9^{\text {th }}$ Interdisciplinary World Congress on Low Back Pain, Singapur, 3. November 2016

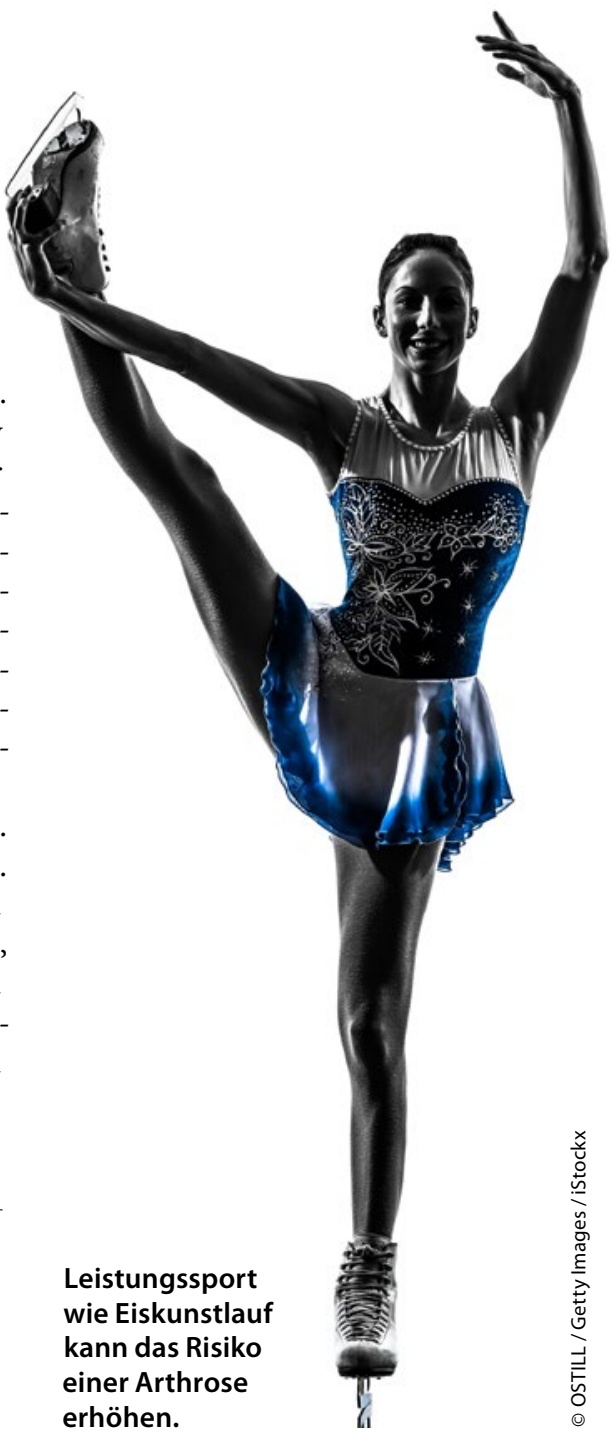

